\title{
Examination of long-term visual memorization capacity in the Clark's nutcracker (Nucifraga columbiana)
}

\author{
Muhammad A. J. Qadri ${ }^{1} \cdot$ Kevin Leonard $^{2} \cdot$ Robert G. Cook ${ }^{1} \cdot$ Debbie M. Kelly ${ }^{2}$ \\ Published online: 15 February 2018 \\ (C) Psychonomic Society, Inc. 2018
}

\begin{abstract}
Clark's nutcrackers exhibit remarkable cache recovery behavior, remembering thousands of seed locations over the winter. No direct laboratory test of their visual memory capacity, however, has yet been performed. Here, two nutcrackers were tested in an operant procedure used to measure different species' visual memory capacities. The nutcrackers were incrementally tested with an ever-expanding pool of pictorial stimuli in a two-alternative discrimination task. Each picture was randomly assigned to either a right or a left choice response, forcing the nutcrackers to memorize each picture-response association. The nutcrackers' visual memorization capacity was estimated at a little over 500 pictures, and the testing suggested effects of primacy, recency, and memory decay over time. The size of this long-term visual memory was less than the approximately 800-picture capacity established for pigeons. These results support the hypothesis that nutcrackers' spatial memory is a specialized adaptation tied to their natural history of food-caching and recovery, and not to a larger long-term, general memory capacity. Furthermore, despite millennia of separate and divergent evolution, the mechanisms of visual information retention seem to reflect common memory systems of differing capacities across the different species tested in this design.
\end{abstract}

Keywords Memory $\cdot$ Comparative cognition $\cdot$ Specialization $\cdot$ Clark's nutcrackers

Clark's nutcrackers (Nucifraga columbiana) are renowned for storing and recovering large numbers of seed caches that are critical to surviving the winter (Tomback, 1980). Nutcrackers can even recover seeds from caches buried in snow that occludes the local visual cues that were present when the seeds were originally cached. This apparently large spatial memory has made nutcrackers of particular comparative interest. Their cache recovery ability is cited regularly as one of the best examples of an evolved specialized cognitive capacity. As a result, this ability ranks among the best evidence supporting an adaptive, as opposed to a generalist, framework for thinking about the evolution and organization of cognition (Balda \& Kamil, 2002; Bitterman, 1975; Shettleworth, 1984; Thorndike, 1898). The latter issue directly connects to the

Muhammad A. J. Qadri

Muhammad.Qadri@tufts.edu

1 Department of Psychology, Tufts University, 490 Boston Avenue, Medford, MA 02155, USA

2 Department of Psychology, University of Manitoba, Winnipeg, Manitoba, Canada longer-standing debate about the organization of human intelligence (Binet, Simon, \& Terman, 1980; Gardner, 1985; Spearman, 1904; Sternberg, 1977; Thurstone, 1924). Further understanding of how cognitive processes diversified in nonhuman species will provide greater perspective on human cognitive evolution.

The cognitive-specialization hypothesis has gained support from numerous spatial-memory studies demonstrating that the cache recovery capacity of nutcrackers exceeds those of other closely related corvids (e.g., Balda \& Kamil, 1989). Operant short-term memory tasks have revealed that nutcrackers can remember spatial information better than visual information (Olson, Kamil, Balda, \& Nims, 1995). Furthermore, wildcaught nutcrackers have enlarged hippocampal formations (Capaldi, Robinson, \& Fahrbach, 1999) or a greater number of neurons in the hippocampus (Basil, Kamil, Balda, \& Fite, 1996; Gould et al., 2013) than less cache-reliant species do (Capaldi et al., 1999).

An alternative account is that these memory abilities are not unique to spatial information or cache recovery, however, but instead reflect the operation of larger, general-purpose memory or cognitive capacity. For instance, Clark's nutcrackers possess many other advanced skills, including abstract 
concept learning (Magnotti, Katz, Wright, \& Kelly, 2015), mirror-self recognition (Clary \& Kelly, 2016), inferential reasoning (Clary \& Kelly, 2013; Tornick \& Gibson, 2013), and situationally specific cache protection strategies (Clary \& Kelly, 2011). Furthermore, corvids in general, though not nutcrackers specifically, have been found to possess superior tool-use abilities (Emery \& Clayton, 2004). These data suggest a greater degree of generalized intelligence, opening the possibility that their renowned spatial memory stems from a larger general-purpose memory system. For the purposes of this article, these two accounts are labeled the specializedmemory and generalized-memory hypotheses, respectively.

Although the long-term memory capacity of nutcrackers has been directly evaluated in the laboratory, generating a sufficient number of controlled caching sites to sufficiently challenge their spatial memory has been difficult (Balda \& Kamil, 1992). The large estimates of their spatial-memory capacity have instead been derived from indirect calculations from natural observations (Balda \& Kamil, 1992). Therefore, whether nutcrackers have a large, specialized spatial memory or simply a larger capacity to store and use information of all types is an open question.

Our understanding and measurement of the long-term memory abilities of several species have recently been advanced using a relatively new testing procedure. The longterm visual memory capacities of two pigeons (Columba livia), two baboons (Papio papio), and one human (Homo sapiens) have been examined using comparable operant procedures (Cook, Levison, Gillett, \& Blaisdell, 2005; Fagot \& Cook, 2006; Voss, 2009). In such procedures, the observer is required to associate randomized right and left responses to an increasingly large pool of successively introduced pictures. The pictures' content is unrelated to their correct choice assignments, so only memorized left-right associations can lead to correct responses. Accuracy with these memorized items over time then provides estimates of the number of simultaneous picture-response associations that can be maintained. For pigeons, a limit of approximately 800 items was found. For baboons and humans, no limit was reached during testing, even after learning 3,500 to 5,000 items for baboons, or 3,400 items for the single human.

This experimental procedure for measuring long-term visual association formation enables the examination of nutcrackers' memory capacity directly and its comparison to the established values for pigeons, baboons, and humans. If, on the one hand, nutcrackers have a large generalized memory capacity, their measured capacity should exceed that of pigeons, and perhaps approach that of primates. This result would be consistent with the generalized-memory account and suggest that nutcrackers are not specifically adapted for cache recovery. If, on the other hand, they possess specialized spatial memory mechanisms devoted just to caching, this operant visual procedure should not tap into that ability. In that case, their measured capacity might look more like that of noncaching pigeons. The latter outcome would be consistent with the specialized-memory account.

Here, two nutcrackers were tested continuously with the same memory procedure and pictures that had previously been used (Cook et al., 2005; Fagot \& Cook, 2006). The primary question of interest was estimating the size of the nutcrackers' visual memory capacity and comparing it to other known results. We additionally examined their speed of learning, choice reaction times (RTs), lag effects, primacy/recency effects, and the proportion of stimuli learned in one trial, in order to examine the operation of the memory mechanisms involved.

\section{Method}

\section{Animals}

Two wild-caught male nutcrackers (Cornelius and Gabby) were tested. Both were naïve to visual operant testing but had participated in spatial cognition studies over the past 12 years. The University of Manitoba's Animal Care Committee approved all procedures (protocol \#F10-029).

\section{Apparatus}

The nutcrackers were tested in a custom-built touchscreen chamber. The stimuli were presented on an LCD monitor (ThinkVision L171p; resolution: $1,024 \times 768$ ) located just behind a $32 \times 27 \mathrm{~cm}$ infrared Open-Frame viewing window (Elo CarrollTouch). A ceiling light illuminated the chamber at all times, except during timeouts. White noise masked any external sounds. A food reward wheel rotated to provide a single mealworm for correct choices. All experimental events were controlled by a computer (Lenovo M600) connected through a USB digital I/O panel (Phidgets Inc.).

All of the picture stimuli were $20 \times 13 \mathrm{~cm}$ in size $(480 \times$ 300 pixels) and were the same ones used in prior tests (Cook et al., 2005; Fagot \& Cook, 2006). The pictures consisted of landscapes, objects, and abstract photography drawn from different commercial image collections and the Internet.

\section{Procedure}

The testing procedures were as similar as possible to those used with baboons and pigeons. Trials started with a peck to a $3.5-\mathrm{cm}$ white circle. This circle was immediately replaced by a centrally located picture stimulus. After five pecks to it, the picture was removed and yellow choice stimuli appeared on the left (a plus sign) and right (a circle) sides of the display. A peck to the correct choice for the preceding picture resulted in a mealworm reward. A peck to the incorrect choice caused the 
trial to repeat after a brief, dark timeout. Such correction trials were excluded from the data analyses, except when computing the lag function. A 5-s intertrial interval was used.

Birds were presented with a 100 -trial daily session, testing a mixture of old and new items. The new-item trials involved two presentations of 30 recently introduced pictures, which were repeated across sessions until the nutcrackers had reached criterion. The correct responses to these stimuli were randomly and permanently assigned to the left or the right choice at their introduction. The learning criterion was $80 \%$ or better performance on the new items in two consecutive sessions. Once this criterion had been attained, this set of pictures was moved to the pool of old items, and a new set of 30 novel pictures was introduced (only 20 new pictures were used for the first 18 sets). Each session's old-item trials were made from randomly selected pictures from the everexpanding pool of previously learned picture-response associations acquired from memorizing the prior sets. This incremental process of adding sets of pictures continued for 399 sessions for Cornelius (who acquired 58 sets prior to an unrelated health issue) and 734 sessions for Gabby (who acquired 76 sets prior to stopping in light of unchanging capacity estimates).

\section{Results}

Both nutcrackers successfully memorized large numbers of picture-response associations over testing. At the end, Cornelius had an old-item pool containing 1,500 pictures, whereas Gabby's pool contained 2,040 pictures. Although both birds had initially started near $90 \%$ accuracy on the old items, accuracy declined as increasingly more sets of items were included. Over the final ten sets of testing, Cornelius (67.5\%, bootstrapped, bias-corrected, and accelerated $95 \%$ confidence interval $\left.\left[\mathrm{CI}_{95 \%}\right]=[65.9 \%, 69.6 \%]\right)$ and Gabby $\left(65.1 \%, \mathrm{CI}_{95 \%}=[63.3 \%, 66.3 \%]\right)$ had lower correctresponse rates to the randomly selected old items from their respective pools of learned stimuli. Figure 1A shows this gradual decline as a function of the number of items in the old-item pool.

Cook et al. (2005) employed a simple all-or-none model of memory to estimate the number of fully memorized items. In this model, pictures are either fully memorized and accessible at the time of choice or are completely inaccessible and require a guess. The number of fully memorized items can then be estimated by determining what proportion of correct responses (i.e., $100 \%$ accuracy) and random guesses (i.e., $50 \%$ accuracy) would yield the observed level of accuracy. This unpretentious model provides a ready metric for evaluating the minimal memorization capacities of an observer. These results are displayed in Fig. $1 \mathrm{~B}$ in 50 -session blocks. The estimated memory set sizes for
A.

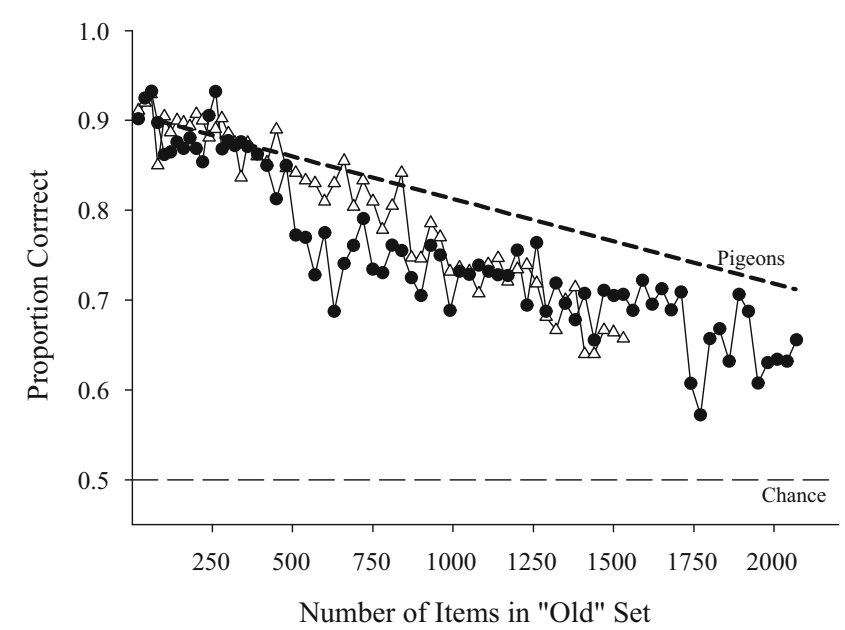

B.

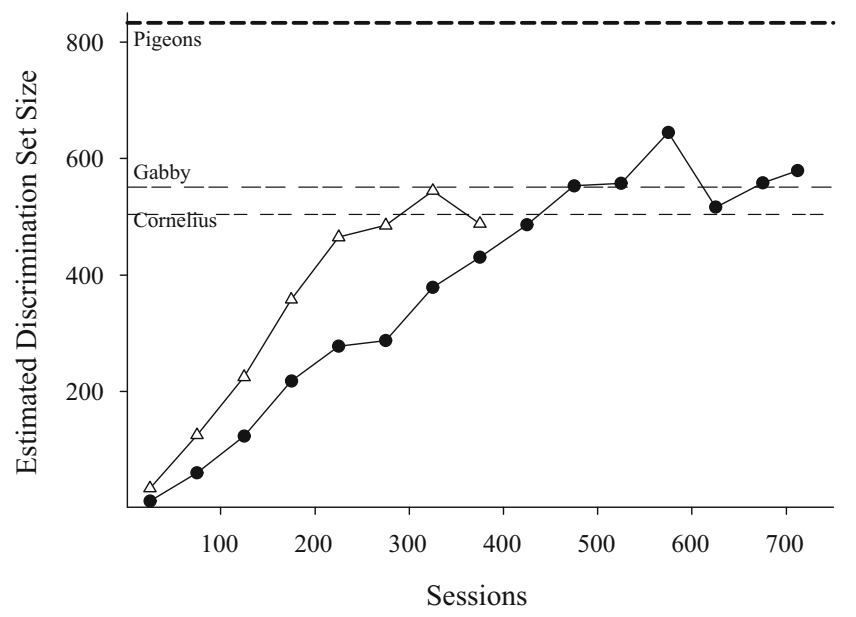

Fig. 1 Proportions correct for memorized items over the experiment as a function of the number of items memorized (A), and estimated memory set size as a function of sessions in the experiment $(\mathbf{B}$; see the text for details of the latter calculation). In both panels, the open triangles are the results for Cornelius, and the filled circles are those for Gabby. The thick dashed lines represent the mean pigeon results from Cook et al. (2005). In panel $\mathbf{A}$, the thin dashed line represents chance performance. In panel $\mathbf{B}$, the thin dashed lines represent the mean estimated discrimination set size estimates from the last three blocks for each bird. Note that Cornelius's and Gabby's last blocks represent only 49 and 34 sessions, respectively, whereas the remaining blocks represent 50 sessions

both nutcrackers steadily increased with additional items, until they eventually plateaued toward the final stages of testing. The estimates over the final three blocks were 504 items $\left(\mathrm{CI}_{95 \%}=[475.9,532.4]\right)$ for Cornelius and 551 items $\left(\mathrm{CI}_{95 \%}=[497.8,595.5]\right)$ for Gabby.

The speed with which the sets of new items were learned stayed constant during the experiment, despite reduced olditem accuracy with increasing memory loads. The numbers of sessions that both subjects completed were each divided into five approximately equal sets for this analysis. The first quintile of the experiment seemed to require slightly more sessions 
for both subjects to learn the first sets of new items, perhaps because of unfamiliarity with operant testing. Averaging the data after the first ten new-item sets revealed that Cornelius averaged about $6.5\left(\mathrm{CI}_{95 \%}=[6.06,6.92]\right)$ sessions to acquire a typical set, whereas Gabby averaged $9.1\left(\mathrm{CI}_{95 \%}=[8.63\right.$, 9.62]) sessions. A mixed-model analysis of these data (fixed effect of quintile, random effect of bird; using the $\mathrm{R}$ package nlme) revealed no significant change during the experiment, $F(1,7)=3.1, p=.123(\alpha=.05$, for this and all remaining tests).

Within the new-item acquisitions, we examined the frequency of items learned with a single exposure (Cook \& Fagot, 2009). Items were designated as "one-trial learning" items if after the first exposure, the bird responded correctly for the next ten exposures (i.e., Exposures 2-11). The nutcrackers learned, on average, $17.3 \%$ of the stimuli with a single exposure (Cornelius: $17.7 \%, \mathrm{CI}_{95 \%}=[15.8,19.6]$; Gabby: $\left.17.0 \%, \mathrm{CI}_{95 \%}=[15.4,18.6]\right)$. Additionally, one-trial learning was more prevalent when the first exposure was rewarded than when it was punished. For Cornelius and Gabby, these occurred at a 2.5:1 rewarded:punished rate $\left(\mathrm{CI}_{95 \%}=[1.94,3.25]: 1\right)$ and a $4.1: 1$ rate $\left(\mathrm{CI}_{95 \%}=[3.10\right.$, 5.26]:1), respectively. The proportions of these one-trial learning items did not change over the course of the experiment (fixed effect of quintile, random effect of item nested within bird, $\chi^{2}(1)=0.6, p>.250$, using the $\mathrm{R}$ package lme4).

The prior comparative research identified that the time between successive stimulus presentations affected accuracy. Figure 2A plots the nutcrackers' accuracy against the number of intervening stimulus presentations (including correction trials) between successive stimulus presentations in the last half of the experiment. Both nutcrackers performed more accurately for recently tested stimuli, with decreasing accuracy as the intervening duration between tests increased (Cornelius slope $=-11.5 \times 10^{-6}, p=.004, R^{2}=.47 ;$ Gabby slope $=-7.65 \times 10^{-}$ $\left.{ }^{6}, p=.009, R^{2}=.63\right)$.

We next examined whether the nutcrackers' memory was susceptible to primacy and recency learning effects. Figures $2 \mathrm{~B}$ and $\mathrm{C}$ address primacy and recency, respectively, by depicting accuracy during the last half of the experiment for the first and last 24 stimulus sets learned, according to their serial position. The earliest sets learned and the most recently learned sets showed high accuracy, and as the level of primacy or recency decreased, accuracy was reduced by approximately ten percentage points. Fitting upward-opening quadratic functions to these data confirmed that the curves display the expected boosts from primacy (Cornelius overall $R^{2}=.54$, Gabby $R^{2}=.61$ ) and recency (Cornelius overall $R^{2}=.91$, Gabby $R^{2}=.76$ ), as compared to items memorized during the middle of the experiment.

Finally, we also evaluated the choice time that the nutcrackers needed to respond to the pictures. For pigeons, this choice time was captured by measuring the latency to the first peck of the picture. This was done because the spatial location of the first peck to the pictures was highly predictive of the final choice. This was also true of the nutcrackers' pecking behavior to the pictures. When examining spatially directed pecking (pecks greater than 50 pixels from the midline of the picture; Cornelius $96.3 \%$ of choices, Gabby $87.7 \%$ ), the side of the first peck reliably predicted the subsequent choice response $90 \%$ of the time (Cornelius $93.1 \%$, Gabby $86.1 \%$ ). Thus, like pigeons, the nutcrackers seemingly had made their choice "decision" by the time of their first peck to the picture. Whereas this choice time measure had decreased over the experiment for pigeons, however, the nutcrackers showed the opposite pattern (Fig. 2D). The first-peck-choice latency slowly increased from near 1,500 ms during the first quintile of the experiment to near $2,000 \mathrm{~ms}$ by the last quintile. A linear mixed-model analysis modeling choice time as a combination of time during the experiment (in quintiles), accuracy, and stimulus set (i.e., old or new item; all as fixed effects with a random effect of subject, calculated using the $\mathrm{R}$ package nlme) confirmed this significant effect of time over the experiment, $F(1,35)=47.8, p<.001$, partial $R^{2}$ for $\operatorname{GLMM}\left(R_{\mathrm{p}}{ }^{2}\right)=$ $.49{ }^{1}$ Accuracy was also a significant predictor of first-peck latency, $F(1,35)=12.2, p=.001, R_{\mathrm{p}}{ }^{2}=.20$, with incorrect responses estimated to be $211 \mathrm{~ms}$ slower than correct responses.

\section{Discussion}

Clark's nutcrackers exhibited overall lower long-term visual memory capacity than has previously been established with pigeons. The estimated asymptotic memory capacity for the two nutcrackers $(\approx 530)$ was less than that found for pigeons $(\approx 830$; see Fig. 1; cf. Cook et al., 2005). Even with measurement error, the nutcrackers' long-term pictorial memory is at best the same as or smaller than pigeons' when tested almost identically. Therefore, the nutcrackers' extraordinary caching and recovery abilities are not due to having a larger generalized memory capacity. If these behaviors were supported by general-purpose memory, the birds' capacity to learn pictureresponse associations should have measurably exceeded those of a noncaching bird like the pigeon. If anything, the pigeons had slightly better general memory. This result offers further support for the specialized-memory hypothesis, in which the cache recovery ability of Clark's nutcrackers is rooted in a memory system that has evolved differentially for storing spatial information relevant to its niche.

\footnotetext{
${ }^{1}$ Proper effect sizes for linear mixed-effect models are currently an active area of applied research. We used the marginal $R_{\text {GLMM }}^{2}$ and report the ratio of the factor's variation accounted for (determined by dropping it from the model) to the sum of the factor's variation and the model's unexplained variation. This parallels the method of computing eta-squared in traditional analyses of variance.
} 


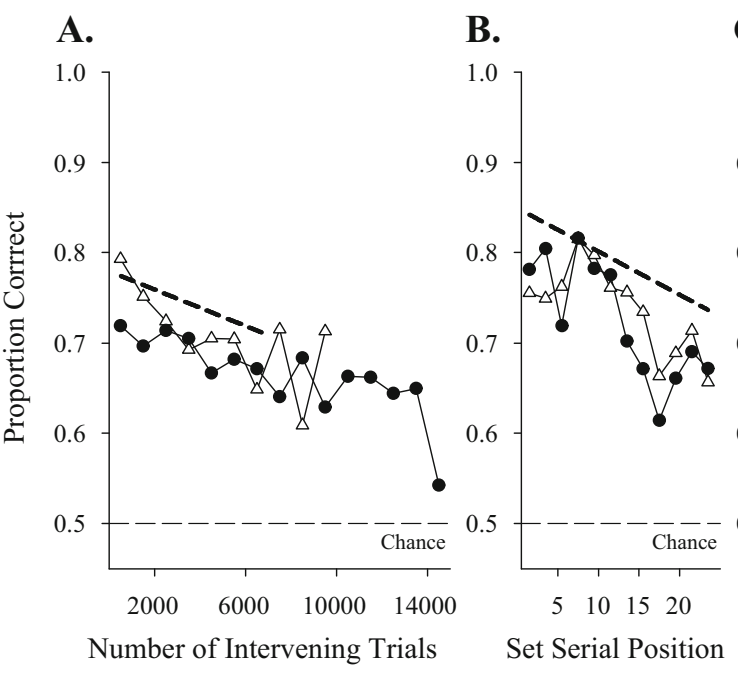

Fig. 2 Proportions correct as a function of the number of intervening trials between presentations during the last half of the experiment (lag; panel A); proportions correct during the last half of the experiment for the first 24 stimulus sets, as a function of the serial position at which they were learned (serial position; panel B); proportions correct during the last half of the experiment for the most recent 24 stimulus sets, as a function of how long ago they were learned (reverse serial position; panel $\mathbf{C}$ ); and latencies to the first peck as a function of the proportion of the experiment

Beyond comparable visual memory capacities, the results in Fig. 2 point to other similarities in the long-term memory systems of these two avian species. Both species show evidence of memory decline, with more recently seen stimuli supporting better accuracy than stimuli with larger test-retest lags. Besides this recency effect, both bird species also show recency and primacy learning effects, in which the earliest and latest sets learned generally support better performance than those learned in between. Additionally, neither species required additional time to learn new sets of pictures, despite increasing memory loads of old items. Given the nature of caching and recovering seeds, we thought that the nutcrackers might show a greater degree of "one-trial learning." The nutcrackers' average of $17.3 \%$ of stimuli learned in one trial, however, is not better than pigeons' $20.8 \%$. These shared properties are good evidence that the memory systems tapped by this task operate in fundamentally similar ways. One possible difference was reflected in choice RTs. For the nutcrackers, RTs increased with extended testing, whereas for the pigeons (and primates) this measure decreased over testing. Whether this reflects differences in memory search mechanisms or other aspects of the task, such as automaticity or procedural learning, is an open question.

Although consistent with the specialized-memory hypothesis, considering other reasons why the nutcrackers were not better than the pigeons is sensible. One possibility is that the pigeons' response (head poke) was separated from the stimulus presentation apparatus (touchscreen), which could have supported stronger spatial representations. Additionally, the pigeons were relatively young and experimentally naïve, in
C. D.

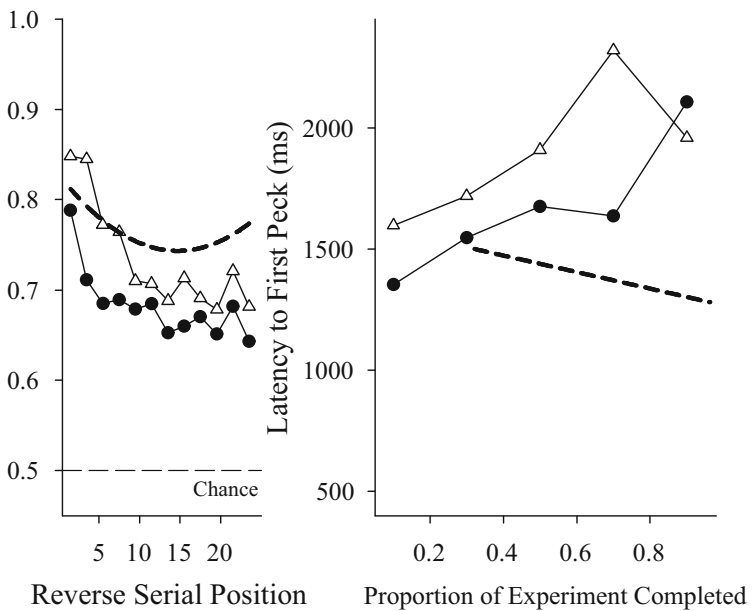

completed (RT; panel D), with corresponding comparisons in each panel to pigeon results (depicted by thick dashed lines; Cook et al., 2005). In all panels, triangles show the results for Cornelius, and the circles are those for Gabby. The thin dashed lines in panels $\mathbf{A}, \mathbf{B}$, and $\mathbf{C}$ represent chance performance. In panel $\mathbf{A}$, although there were instances of more than 14,000 intervening trials for Gabby and more than 10,000 for Cornelius, these data are omitted because of their sparsity

contrast to the nutcrackers, who had been in the laboratory for 12 years. Beyond possible cognitive effects of aging, one study has shown that chickadees' hippocampus size (and therefore, presumably, function) diminishes with less than two months of captivity (Tarr, Rabinowitz, Imtiaz, \& DeVoogd, 2009). In the absence of any natural caching behavior during their captivity (during which, however, they had participated in spatial memory experiments), our nutcrackers' capacity for memorizing large amounts of information, visual or not, might have diminished. Another possibility lies in the task. Because the stimulus information is spatially irrelevant and the choice outputs are spatially redundant and highly repetitive, the spatial parts of the task are not as varied as during caching in the wild. When caching, contexts and sequences may aid visual memory for cache locations, and the variability of spatial response locations may greatly reduce proactive interference (Lewis \& Kamil, 2006; Olson et al., 1995). Finally, nutcrackers choose their caching locations in the wild, which may result in a "generation effect" that boosts memory (Kornell \& Terrace, 2007; Slamecka \& Graf, 1978). These factors were unavailable here. Perhaps if more spatial response outputs or different encoding contexts had been provided, the nutcrackers might have exhibited more robust and generalized visuospatial memory abilities. Of course, the same argument would hold for the pigeons, who shared these same potential disadvantages.

Nonetheless, this evidence indicates that the archetypal caching species' memory does not rival that of a common, noncaching bird species. When we compare the four species now tested on this memory task (humans, baboons, pigeons, 
and nutcrackers), the basic operation of their retention mechanisms seems substantially to be conserved. Despite millennia of separate and divergent evolution, the mechanisms of visual information retention in each species seems to reflect the common operation of a large durable memory system that can be searched in parallel, with the most frequently repeated or most recent items being retained best. The only clear difference is that the two primate species have a several-fold larger pictorial memory capacity than the two avian species.

These findings contribute to the ongoing debate about the nature of human and nonhuman animal intelligence. Although definitions of intelligence vary, the results indicate that longterm memory capacity is not strongly related to complex cognition. Presumably, a larger memory store might permit greater retention of more experience and information and allow the abstract relations among items or subsets of items to be discovered or connected. The notion that corvids have more cognitive flexibility than pigeons has wide support. For example, corvids consistently solve more complex problems in the laboratory than do pigeons (Bluff, Weir, Rutz, Wimpenny, \& Kacelnik, 2007; Magnotti et al., 2015). The same is true in comparisons of human and baboon problem solving. Yet as we measured in the present memory task, neither of these more "advanced" problem-solving species exhibited dramatically greater memory capacity than its less "advanced" brethren. This suggests that problem solving, cognitive flexibility, and intelligence reside elsewhere than in the long-term memory organization of the avian and primate nervous systems.

Author note This research was supported by grants from the National Eye Institute (\#RO1EY022655, to R.G.C.) and the Natural Sciences \& Engineering Research Council of Canada (\#312379-2009, to D.M.K.).

\section{References}

Balda, R. P., \& Kamil, A. C. (1989). A comparative study of cache recovery by three corvid species. Animal Behaviour, 38, 486-495. https://doi.org/10.1016/S0003-3472(89)80041-7

Balda, R. P., \& Kamil, A. C. (1992). Long-term spatial memory in Clark's nutcracker, Nucifraga columbiana. Animal Behaviour, 44, 761-769. https://doi.org/10.1016/S0003-3472(05)80302-1

Balda, R. P., \& Kamil, A. C. (2002). Spatial and social cognition in corvids: An evolutionary approach. In M. Bekoff, C. Allen, \& G. M. Burghardt (Eds.), The cognitive animal: Empirical and theoretical perspectives on animal cognition (pp. 129-133). Cambridge, MA: MIT Press.

Basil, J. A., Kamil, A. C., Balda, R. P., \& Fite, K. V. (1996). Differences in hippocampal volume among food storing corvids. Brain, Behavior and Evolution, 47, 156-164. https://doi.org/10.1159/ 000113235

Binet, A., Simon, T., \& Terman, L. M. (1980). The development of intelligence in children. Nashville, TN: Williams.

Bitterman, M. E. (1975). The comparative analysis of learning. Science, 188, 699-709. https://doi.org/10.1126/science.188.4189.699

Bluff, L. A., Weir, A. A. S., Rutz, C., Wimpenny, J. H., \& Kacelnik, A. (2007). Tool-related cognition in new Caledonian crows.
Comparative Cognition and Behavior Reviews, 2, 1-25. https:// doi.org/10.3819/ccbr.2008.20001

Capaldi, E. A., Robinson, G. E., \& Fahrbach, S. E. (1999). Neuroethology of spatial learning: The birds and the bees. Annual Review of Psychology, 50, 651-682. https://doi.org/10.1146/ annurev.psych.50.1.651

Clary, D., \& Kelly, D. M. (2011). Cache protection strategies of a nonsocial food-caching corvid, Clark's nutcracker (Nucifraga columbiana). Animal Cognition, 14, 735-744. https://doi.org/10. 1007/s10071-011-0408-3

Clary, D., \& Kelly, D. M. (2013). Are Clark's nutcrackers (Nucifraga columbiana) able to discriminate knowledge states of human experimenters during an object-choice task? Evolutionary Psychology, 11, 147470491301100310 . https://doi.org/10.1177/ 147470491301100310

Clary, D., \& Kelly, D. M. (2016). Graded mirror self-recognition by Clark's nutcrackers. Scientific Reports, 6, 36459. https://doi.org/ 10.1038/srep36459

Cook, R. G., \& Fagot, J. (2009). First trial rewards promote 1-trial learning and prolonged memory in pigeon and baboon. Proceedings of the National Academy of Sciences, 106, 9530-9533. https://doi.org/ 10.1073/pnas.0903378106

Cook, R. G., Levison, D. G., Gillett, S. R., \& Blaisdell, A. P. (2005). Capacity and limits of associative memory in pigeons. Psychonomic Bulletin \& Review, 12, 350-358. https://doi.org/10.3758/ BF03196384

Emery, N. J., \& Clayton, N. S. (2004). The mentality of crows: Convergent evolution of intelligence in corvids and apes. Science, 306, 1903. https://doi.org/10.1126/science.1098410

Fagot, J., \& Cook, R. G. (2006). Evidence for large long-term memory capacities in baboons and pigeons and its implication for learning and the evolution of cognition. Proceedings of the National Academy of Science, 103, 17564-17567. https://doi.org/10.1073/ pnas.0605184103

Gardner, H. (1985). Frames of mind: The theory of multiple intelligences. New York, NY: Basic Books.

Gould, K. L., Gilbertson, K. E., Hrvol, A. J., Nelson, J. C., Seyfer, A. L., Brantner, R. M., \& Kamil, A. C. (2013). Differences in relative hippocampus volume and number of hippocampus neurons among five corvid species. Brain, Behavior and Evolution, 81, 56-70. https://doi.org/10.1159/000345560

Kornell, N., \& Terrace, H. S. (2007). The generation effect in monkeys. Psychological Science, 18, 682-685. https://doi.org/10.1111/j.14679280.2007.01959.x

Lewis, J. L., \& Kamil, A. C. (2006). Interference effects in the memory for serially presented locations in Clark's nutcrackers, Nucifraga columbiana. Journal of Experimental Psychology: Animal Behavior Processes, 32, 407-418. https://doi.org/10.1037/00977403.32.4.407

Magnotti, J. F., Katz, J. S., Wright, A. A., \& Kelly, D. M. (2015). Superior abstract-concept learning by Clark's nutcrackers (Nucifraga columbiana). Biology Letters, 11, 148. https://doi.org/10.1098/rsbl. 2015.0148

Olson, D. J., Kamil, A. C., Balda, R. P., \& Nims, P. J. (1995). Performance of four-seed caching corvid species in operant tests of nonspatial and spatial memory. Journal of Comparative Psychology, 109, 173-181.

Shettleworth, S. J. (1984). Natural history and evolution of learning in nonhuman mammals. In P. Marler \& H. S. Terrace (Eds.), The biology of learning: Report of the Dahlem Workshop on the Biology of Learning Berlin, 1983, October 23-28 (pp. 419-433). Berlin, Germany: Springer Berlin Heidelberg.

Slamecka, N. J., \& Graf, P. (1978). The generation effect: Delineation of a phenomenon. Journal of Experimental Psychology: Human Learning and Memory, 4, 592-604. https://doi.org/10.1037/02787393.4.6.592 
Spearman, C. (1904). "General intelligence," objectively determined and measured. American Journal of Psychology, 15, 201-292. https:// doi.org/10.2307/1412107

Sternberg, R. J. (1977). Intelligence, information processing, and analogical reasoning: The componential analysis of human abilities. Hillsdale, NJ: Erlbaum.

Tarr, B. A., Rabinowitz, J. S., Imtiaz, M. A., \& DeVoogd, T. J. (2009). Captivity reduces hippocampal volume but not survival of new cells in a food-storing bird. Developmental Neurobiology, 69, 972-981. https://doi.org/10.1002/dneu.20736

Thorndike, E. L. (1898). Animal intelligence: An experimental study of the associative processes in animals. Psychological Monographs: General and Applied, 2(4). https://doi.org/10.1037/10780-000
Thurstone, L. L. (1924). The nature of intelligence. London, UK: Routledge.

Tomback, D. F. (1980). How nutcrackers find their seed stores. Condor, $82,10-19$.

Tornick, J. K., \& Gibson, B. M. (2013). Tests of inferential reasoning by exclusion in Clark's nutcrackers (Nucifraga columbiana). Animal Cognition, 16, 583-597. https://doi.org/10.1007/s10071-013-0595-1

Voss, J. L. (2009). Long-term associative memory capacity in man. Psychonomic Bulletin \& Review, 16, 1076-1081. https://doi.org/ 10.3758/pbr.16.6.1076 